\title{
Réponse immunitaire sérique chez les patients rétablis de la COVID-19 dont le résultat à un test subséquent est positif
}

\author{
Nicole Atchessi ${ }^{1}$, Megan Striha ${ }^{1}$, Rojiemiahd Edjoc ${ }^{1 *}$, Christine Abalos ${ }^{1}$, Amanda Lien ${ }^{1}$, \\ Lisa Waddell2, Imran Gabrani-Juma', Emily Thompson', Thomas Dawson ${ }^{1}$
}

\section{Résumé}

Contexte : Des études comparant la réponse immunitaire de patients rétablis de la maladie à coronavirus 2019 (COVID-19) dont le résultat à un test subséquent a été positif (TP) en utilisant la réaction en chaîne par polymérase après transcription inverse (RT-PCR) et celle de patients qui ont obtenu un résultat négatif $(T N)$ à un test subséquent ont été utilisées pour étudier une relation possible entre la réponse immunitaire et le statut du nouveau test.

Méthodes : Sept bases de données ont été consultées. Les critères de recherche comprenaient les études de cohorte et les études cas-témoins réalisées dans le monde entier et publiées avant le 9 septembre 2020. Ces études comparaient les taux sériques d'anticorps des cas de patients atteints de COVID-19 hospitalisés dont le résultat à un test après leur sortie de I'hôpital a été positif (TP) à ceux dont le résultat au test a été négatif (TN).

Résultats : Certaines données indiquent que les taux d'immunoglobuline G (lgG) et d'immunoglobuline $M(\lg M)$ étaient plus faibles chez les patients dont le résultat à un test subséquent était positif que chez les patients dont le résultat au nouveau test était négatif. L'hypothèse d'une clairance incomplète cadre avec ces résultats. La possibilité d'obtenir des faux négatifs à l'épreuve d'amplification en chaîne par RT-PCR pendant la clairance virale est également plausible, car la concentration d'acide ribonucléique (ARN) viral dans les écouvillons nasopharyngés et fécaux fluctue en dessous des limites de détection de la RT-PCR pendant la clairance virale. La probabilité d'une réinfection était moins susceptible d'être la cause d'un nouveau résultat positif en raison du faible risque d'exposition lorsque les patients observaient une quarantaine de 14 jours après leur sortie de I'hôpital.

Conclusion : D'autres études sont nécessaires pour mieux expliquer la réponse immunitaire des cas de patients rétablis de la COVID-19 dont le résultat à un test est positif après leur sortie de I’hôpital.
Cette oeuvre est mise à la disposition selon les termes de la licence internationale Creative Commons Attribution 4.0

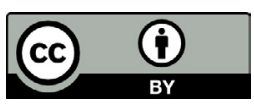

Affiliations

${ }^{1}$ Direction générale de la sécurité sanitaire et des opérations, Agence de la santé publique du Canada, Ottawa, ON

${ }^{2}$ Division des sciences du risque en santé publique, Laboratoire national de microbiologie, Agence de la santé publique du Canada, Winnipeg, MB

\section{*Correspondance :}

rojiemiahd.edjoc@canada.ca

Citation proposée : Atchessi N, Striha M, Edjoc R, Abalos C, Lien A, Waddell L, Gabrani-Juma I, Thompson $E$, Dawson T. Réponse immunitaire sérique chez les patients rétablis de la COVID-19 dont le résultat à un test subséquent est positif. Relevé des maladies transmissibles au Canada 2021;47(4):213-20.

https://doi.org/10.14745/ccdr.v47i04a03f

Mots-clés : COVID-19, SRAS-CoV-2, RT-PCR, faux négatifs, réinfection, nouvelle analyse

\section{Introduction}

La maladie à coronavirus 2019 (COVID-19) est une nouvelle maladie qui résulte d'une infection par le coronavirus du syndrome respiratoire aigu sévère 2 (SRAS-CoV-2) (1). Le 31 décembre 2019, un cas de pneumonie de cause inconnue a été identifié à Wuhan, en Chine, et signalé au bureau de pays de l'Organisation mondiale de la Santé (OMS). II a été confirmé par la suite que la cause de la maladie était un nouveau coronavirus (2). L'éclosion du SRAS-CoV-2 a été déclarée pandémie par l'OMS le 11 mars 2020 (3). En date du 14 octobre 2020, $75 \%$ des 38508475 de cas de COVID-19 confirmés à l'échelle mondiale se sont rétablis (4). Cependant, les faits démontrent que certains cas de COVID-19 rétablis, sortis de l'hôpital avec des résultats négatifs au dépistage de la COVID-19, ont ensuite obtenu un résultat positif à un test de dépistage subséquent (5). 


\section{Situation actuelle}

Le fait que les cas de COVID-19 rétablis ou sortis de l'hôpital obtiennent un résultat positif à un nouveau test soulève des inquiétudes quant à une réinfection potentielle ou à une clairance virale incomplète, ainsi que des questions liées à I'infectiosité du patient. De multiples hypothèses ont été avancées pour expliquer les raisons pour lesquelles certains cas de patients obtiennent un résultat positif à un nouveau test une fois sortis de l'hôpital avec des résultats négatifs au test de réaction en chaîne par polymérase après transcription inverse (RT-PCR). L'objectif de cette revue est d'explorer les données actuelles concernant les différences de réponse immunitaire chez les patients atteints de COVID-19 sortis de I'hôpital dont le résultat au nouveau dépistage est positif (TP) et ceux dont le test est négatif (TN).

\section{Méthodes}

Nos critères de recherche comprenaient les études de cohorte et les études cas-témoins réalisées dans le monde entier et publiées avant le 9 septembre 2020 qui comparaient le taux sérique d'anticorps des cas de COVID-19 hospitalisés dont le résultat à un nouveau dépistage était positif après leur sortie de I'hôpital, à ceux dont le test était négatif. Sept bases de données ont été consultées. Les termes de recherche utilisés étaient les suivants : réactivation, réinfection, réapparition, récurrence, en conjonction avec hospitalisation, sortie de l'hôpital, anticorps et immunité. Les articles $(n=224)$ ont été sélectionnés en raison de leur pertinence, et les études incluses ont été évaluées à l'aide de l'outil d'évaluation du risque de biais de l'échelle de Newcastle-Ottawa selon des critères liés à la sélection des cas (score $=4)$, à la comparabilité des cas dont le test subséquent était positif et des cas dont le test était négatif (score $=2$ ), et à l'évaluation de l'exposition ou du résultat (score $=3$ ). Au total, neuf études ont été jugées pertinentes (voir annexe A). Un suivi d'au moins quatre semaines a été considéré comme étant adéquat dans les études de cohorte. L'évaluation a été effectuée par deux examinateurs (voir annexe B).

\section{Résultats}

Nous avons déterminé trois études de cohorte prospectives (6-8), trois études de cohorte rétrospectives (9-11), deux études de cohorte (dont on ne sait pas si elles sont prospectives ou rétrospectives) $(12,13)$ et une étude cas-témoins (14).

La taille de l'échantillon des neuf études variait entre 74 et 619. La prévalence des cas de COVID-19 qui ont connu une réapparition d'un TP après avoir rempli les critères de sortie consistant en deux tests RT-PCR négatifs consécutifs à plus de 24 heures d'intervalle, variait de $7 \%$ à $21 \%$ dans sept des huit études de cohorte $(6-8,10-14)$. La neuvième étude a trouvé $58 \%$ de cas avec un TP (9). La prévalence moyenne pour les huit études de cohorte était de $16,6 \%$ ( $n=382 / 2305$ cas). Parmi les six études qui ont recueilli des informations sur le temps écoulé entre le test négatif à la sortie de l'hôpital et le TP au premier dépistage après la sortie, le délai médian variait de 4,6 jours à 12 jours $(6,8,10,11,13,14)$. Les cas de TP subséquents étaient significativement plus jeunes dans quatre études $(6,8,13,14)$; cependant, aucune différence d'âge n'a été constatée dans les cinq autres études $(7,9-12)$. Une association avec le sexe n'a été rapportée dans aucune des études.

Six études ont fait état du taux de positivité des anticorps anti-immunoglobuline $\mathrm{G}$ (lgG) et anti-immunoglobuline $\mathrm{M}$ ( $\mathrm{lgM}$ ) $(7,8,10-13)$, tandis que quatre ont précisé leurs niveaux sériques $(8,9,12,14)$. II n'y avait pas de différence dans les taux de positivité des anticorps lgG et lgM entre les cas TP et les TN $(6-8,10-13)$; cependant, le niveau d'anticorps IgG $(9,14)$ ou lgM $(8,14)$ dans le sérum était plus faible chez les cas de patients TP comparé aux patients TN dans trois des études. Aucune différence n'a été observée dans les autres études concernant le taux $d^{\prime} \lg M(9,12)$ ou d'lgG $(8,12)$.

\section{Discussion}

II existe plusieurs explications possibles pour les cas de résultat positif à un nouveau test après la sortie de l'hôpital, comme une faible charge virale dans les échantillons, des faux négatifs aux tests RT-PCR et une réinfection. L'explication la plus probable est un résultat RT-PCR faussement négatif dû 1) à la concentration d'acide ribonucléique (ARN) viral diminuant jusqu'à des niveaux inférieurs à la limite de détection du test pendant la clairance virale et 2) à la proportion attendue de faux négatifs dans les tests RT-PCR.

Selon Liu et al. (12), étant donné que la présence d'anticorps IgM était similaire dans les cas de TP subséquents et de TN subséquents, les résultats nouvellement positifs aux tests RT-PCR dans leur étude de 619 cas étaient peu susceptibles d'être dus à une réinfection par le virus SRAS-CoV-2. Les études incluses dans cette revue ont principalement suivi les cas pendant la période de quarantaine de 14 jours après la sortie de l'hôpital, ce qui exclut également la réinfection comme explication probable des résultats positifs à un nouveau test peu après la sortie de l’hôpital.

Selon Yang et al., les faux négatifs de la RT-PCR peuvent également être dus à de faibles concentrations de virus dans les échantillons provenant d'écouvillons nasopharyngés ou fécaux, alors que le virus est encore présent dans les voies respiratoires inférieures, ce qui entraîne une excrétion intermittente ou fluctuante d'ARN viral dans les voies respiratoires supérieures (8).

Wölfel et al. ont signalé que la RT-PCR pour le SRAS-CoV-2 est un test imparfait, avec une sensibilité de $89 \%$ et, par conséquent, il faut s'attendre à $11 \%$ de faux négatifs (15). Zou (11) a constaté que la réalisation de trois tests consécutifs avant la sortie de l'hôpital réduisait de manière importante 
le risque d'un test positif subséquent, ce qui est conforme à I'hypothèse des faux négatifs.

Les résultats qui établissent un lien entre un niveau plus faible $d$ 'anticorps et un nouveau TP suggèrent que les cas de nouveau TP étaient plus susceptibles d'avoir une réponse immunitaire plus faible, ce qui correspond à l'hypothèse d'une clairance virale. Selon Yuan (7), la plus faible prévalence de tests positifs subséquents chez les personnes présentant des symptômes graves par rapport à celles présentant des symptômes légers peut s'expliquer par une réponse immunitaire plus forte activée chez les personnes gravement malades qui élimine le virus plus efficacement.

L'une des principales préoccupations que suscite un résultat positif à un test subséquent est l'infectiosité des cas après leur sortie de l'hôpital. Alors que certains auteurs affirment que le risque de contagion pendant cette période est faible $(8,16)$, d'autres affirment le contraire et mentionnent même la possibilité d'une infection chronique par le SRAS-CoV-2 (12). Cette dernière hypothèse remet en question le rôle protecteur des anticorps lgG et l'utilisation des tests sérologiques pour établir l'immunité.

Les résultats de cette étude semblent confirmer l'existence d'une association entre la réponse immunitaire et un nouveau test positif après la sortie de l'hôpital. II existe des données selon lesquelles les taux d'anticorps lgG et lgM sont plus faibles dans les cas de TP subséquents que dans les cas de TN subséquents. L'hypothèse d'une clairance incomplète cadre également avec ces résultats.

\section{Limites}

La présente revue de littérature comporte plusieurs limites. Cinq des neuf études avaient une taille d'échantillon inférieure à 200 et toutes les études étaient limitées à la Chine, ce qui limite la représentativité de la revue de littérature. Nous n'avons pas pu trouver de recherche en dehors de la Chine qui avait des résultats sérologiques, un groupe de comparaison et un test RT-PCR de suivi pour établir le statut de nouveau TP. Parmi les études incluses, la durée du suivi après la sortie de l'hôpital et les intervalles entre les tests RT-PCR était variable. Cela aurait pu avoir un impact sur les résultats en fonction du moment de leur réalisation et sur la prévalence des TP résumés dans cette étude. En outre, huit études sur neuf étaient fondées sur des cas de sortie d'hôpital, mais aucune information sur leur représentativité des cas de patients hospitalisés n'a été fournie. De plus, l'absence de comparaison entre les cas dont les dossiers médicaux étaient incomplets ou perdus de vue et ceux qui ont poursuivi les études limite l'évaluation de biais potentiels. Cette revue de littérature inclut des études publiées jusqu'en septembre 2020 et doit être interprétée en conséquence, étant donné l'évolution rapide des données. II pourrait être intéressant pour les études futures de se concentrer sur les méthodes de dépistage ayant une faible probabilité de faux négatifs afin de s'assurer que les cas de COVID-19 hospitalisés répondent aux critères requis avant leur sortie.

Aucune des études n'a utilisé une analyse multivariée pour déterminer les associations ajustées des résultats de test avec la réponse immunitaire après contrôle des facteurs de confusion potentiels.

\section{Conclusion}

La situation des cas de tests positifs subséquents pour la COVID-19 après avoir eu deux résultats négatifs au test RT-PCR $n$ 'est pas rare. Des données suggèrent une relation entre les cas de TP subséquents et l'âge $(6,8,13,14)$ et peut-être entre les cas de TP subséquents et la gravité de la maladie. Cependant, aucune de ces études n'a examiné l'association potentielle dans une analyse multivariée avec la réponse immunitaire pour déterminer les associations ajustées après contrôle des facteurs de confusion potentiels. Un travail supplémentaire de synthèse des données avec des études observationnelles appropriées sur les caractéristiques des cas COVID-19 qui ont obtenu un résultat positif à un nouveau test est nécessaire pour mieux comprendre qui est susceptible d'obtenir un résultat positif à un nouveau test. De même, des travaux de recherche et de synthèse supplémentaires sur la réponse immunitaire et l'immunité sont nécessaires pour améliorer notre compréhension de l'infection par la COVID-19.

Les preuves résumées dans ce rapport peuvent avoir des implications importantes pour la santé publique et la gestion des cas de COVID-19 rétablis. Un nombre limité d'études répondait aux critères d'inclusion; cependant, les données suggèrent que la réponse immunitaire dans les cas de COVID-19 convalescents peut être associée à la clairance virale incomplète. Ces résultats préliminaires peuvent être utilisés pour éclairer les recherches ultérieures ou la prise de décision sur ce sujet.

\section{Déclaration des auteurs}

N. A. - Méthodologie, enquête, rédaction-version originale, révision et édition

M. S. - Méthodologie, enquête, rédaction-version originale, révision et édition

R. E. - Conceptualisation, méthodologie, enquête, rédactionprojet original, révision et édition, supervision

C. A. - Rédaction-projet initial, révision et édition

A. L. - Rédaction-projet initial, révision et édition

L. W. - Rédaction-révision et édition

I. G. J. - Rédaction-révision et édition

E. T.- Rédaction-révision et édition

T. D. - Rédaction-révision et édition

\section{Intérêts concurrents}

Aucun intérêt concurrent à déclarer. 


\section{Remerciements}

Nous tenons à remercier nos collègues du Groupe des sciences émergentes de l'Agence de la santé publique du Canada pour leur soutien dans ce travail.

\section{Financement}

Aucun intérêt concurrent à déclarer.

\section{Références}

1. Wu Z, McGoogan JM. Characteristics of and Important Lessons From the Coronavirus Disease 2019 (COVID-19) Outbreak in China: Summary of a Report of 72314 Cases From the Chinese Center for Disease Control and Prevention. JAMA 2020;323(13):1239-42. DOI PubMed

2. Organisation mondiale de la Santé. Allocution liminaire du Directeur général de l'OMS lors de la réunion d'information pour les missions sur la COVID-19 - 9 avril 2020. Genève, Suisse : OMS; 2020 (accédé 2020-05-08). https://www.who. int/fr/director-general/speeches/detail/who-director-generals-opening-remarks-at-the-mission-briefing-on-covid-19---9april-2020

3. Organisation mondiale de la Santé. Allocution liminaire du Directeur général de l'OMS lors du point presse sur la COVID-19 - 11 mars 2020. Genève, Suisse : OMS; 2020 (accédé 2020-09-04). https://www.who.int/fr/director-general/ speeches/detail/who-director-general-s-opening-remarks-atthe-media-briefing-on-covid-19---11-march-2020

4. Worldometer. COVID-19 Coronavirus pandemic. Worldometer; 2020 (accédé 2020-10). https://www.worldometers.info/ coronavirus/

5. Lan L, Xu D, Ye G, Xia C, Wang S, Li Y, Xu H. Positive RT-PCR Test Results in Patients Recovered From COVID-19. JAMA 2020;323(15):1502-3. DOI PubMed

6. Lu J, Peng J, Xiong Q, Liu Z, Lin H, Tan X, Kang M, Yuan R, Zeng L, Zhou P, Liang C, Yi L, du Plessis L, Song T, Ma W, Sun J, Pybus OG, Ke C. Clinical, immunological and virological characterization of COVID-19 patients that test re-positive for SARS-CoV-2 by RT-PCR. EBioMedicine 2020;59:102960. DOI PubMed

7. Yuan B, Liu HQ, Yang ZR, Chen YX, Liu ZY, Zhang K, Wang C, Li WX, An YW, Wang JC, Song S. Recurrence of positive SARS-CoV-2 viral RNA in recovered COVID-19 patients during medical isolation observation. Sci Rep 2020;10(1):11887. DOI PubMed

8. Yang $C$, Jiang $M$, Wang $X$, Tang $X$, Fang $S$, Li $H$, Zuo $L$, Jiang $Y$, Zhong $Y$, Chen $Q$, Zheng $C$, Wang $L$, Wu S, Wu W, Liu H, Yuan J, Liao X, Zhang Z, Shi X, Geng Y, Zhang H, Zheng H, Wan M, Lu L, Ren X, Cui Y, Zou X, Feng T, Xia J, Yang R, Liu Y, Mei S, Li $B$, Yang Z, Hu Q. Viral RNA level, serum antibody responses, and transmission risk in recovered COVID-19 patients with recurrent positive SARS-CoV-2 RNA test results: a population-based observational cohort study. Emerg Microbes Infect 2020;9(1):2368-78. DOI PubMed
9. Hu Q, Cui X, Liu X, Peng B, Jiang J, Wang X, Li Y, Hu W, Zo Z, Duan J, Wang X, Zhu L, Gu G, Guo S. The production and clinical implications of SARS-CoV-2 antibodies. medRxiv. 2020.04.20.20065953. DOI

10. Zhu $H$, Fu L, Jin $Y$, Shao J, Zhang $S$, Zheng $N$, Fan $L$, Yu Z, Ying J, Hu Y, Chen T, Chen Y, Chen M, Chen M, Xiong Z, Kang J, Jin J, Cai T, Ye H. Clinical features of COVID-19 convalescent patients with re-positive nucleic acid detection. J Clin Lab Anal 2020;34(7):e23392. DOI PubMed

11. Zou Y, Wang B-R, Sun L, Xu S, Kong Y-G, Shen L-J, Liang G-T, Chen S-M. The issue of recurrently positive patients who recovered from COVID-19 according to the current discharge criteria: investigation of patients from multiple medical institutions in Wuhan, China. J Infect Dis 2020;222(11);1784-8. DOI

12. Liu T, Wu S, Zeng G, Zhou F, Li Y, Guo F, Wang X. Recurrent positive SARS-CoV-2: immune certificate may not be valid. J Med Virol 2020;92(11):2384-6. DOI PubMed

13. Huang J, Zheng L, Li Z, Hao S, Ye F, Chen J, Yao X, Liao J, Wang S, Zeng M, Qiu L, Cen F, Huang Y, Zhu T, Xu Z, Ye M, Yang $Y$, Wang G, Li J, Wang L, Qu J, Yuan J, Zheng W, Zhang Z, Li C, Whitin JC, Tian L, Chubb H, Hwa K-Y, Gans HA, Ceresnak SR, Zhang W, Lu Y, Maldonado YA, He Q, Wang Z, Liu Y, McElhinney BD, Sylvester KG, Cohen HL, Liu L, Ling XB. Recurrence of SARS-CoV-2 PCR positivity in COVID-19 patients: a single center experience and potential implications. medRxiv. 2020.05.06.20089573. DOI

14. Chen L, Zhang Z-Y, Zhang $X$, Zhang S-Z, Han Q-Y, Feng Z-P, Fu JG, Xiao X, Chen HM, Liu L-L, Chen X-L, Lan Y-P, Zhong D-J, Hu L, Wang J-H, Yin Z-Y. Clinical Characteristics of Recurrent-positive Coronavirus Disease 2019 after Curative Discharge: a retrospective analysis of 15 cases in Wuhan China. medRxiv. 2020.07.02.20144873. DOI

15. Kim $\mathrm{H}$, Hong $\mathrm{H}$, Yoon $\mathrm{SH}$. Diagnostic Performance of CT and Reverse Transcriptase Polymerase Chain Reaction for Coronavirus Disease 2019: A Meta-Analysis. Radiology 2020;296(3):E145-55. DOI PubMed

16. Wölfel R, Corman VM, Guggemos W, Seilmaier M, Zange $S$, Müller MA, Niemeyer D, Jones TC, Vollmar P, Rothe C, Hoelscher M, Bleicker T, Brünink S, Schneider J, Ehmann R, Zwirglmaier K, Drosten C, Wendtner C. Virological assessment of hospitalized patients with COVID-2019. Nature 2020;581(7809):465-9. DOl PubMed 


\section{Annexes}

\section{Annexe A : Caractéristiques sérologiques des cas de maladie à coronavirus 2019 hospitalisés qui ont eu un nouveau test d'amplification en chaîne par polymérase par transcription inverse positif par rapport à ceux dont le nouveau test était négatif}

\section{Description de} l'étude

(référence et type,

lieu et dates de l'étude)

Détails du rapport Principales conclusions et limites
Lu et al., 2020 (6)

Étude de cohorte

Guangdong, Chine Du 23 janvier au 19 février 2020 prospective

- Cette étude a suivi 619 cas sortis de l'hôpital et la sérologie était le principal résultat : 288 cas ont subi un test sérologique 35 jours en moyenne après l'apparition des symptômes (fourchette de 23 à 47 jours).

- Les cas ont été suivis pendant 66 jours après la sortie de I'hôpital, et le nouveau test positif a eu lieu le jour 10 en moyenne (testés aux jours 7 et 14 seulement).

Yang et al., 2020 (8) (préimpression) Étude de cohorte prospective Shenzhen, Chine

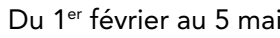
2020

Yuan et al., 2020 (7)

Étude de cohorte prospective

Shenzhen, Chine Avant le 21 avril 2020
- Cette étude a suivi 479 cas sortis de l'hôpital. La sérologie est le principal résultat, les échantillons de sérum étant recueillis les $1^{\mathrm{er}}, 3^{\mathrm{e}}, 7^{\mathrm{e}}$ et $14^{\mathrm{e}}$ jours après la sortie de l'hôpital.

- Les cas ont été suivis pendant 90 jours après leur sortie de l'hôpital et ont obtenu un nouveau test positif le huitième jour en moyenne. Une moyenne de 46 jours s'est écoulée entre le début de la maladie et le dernier résultat positif subséquent pour chaque patient.

- Les titres d'anticorps neutralisants pour les cas de nouveau test positif et de nouveau test négatif n'étaient pas significativement différents 14 jours après la sortie de l'hôpital.

- Cette étude comportait la plus grande cohorte, suivie pendant la plus longue période, et a enregistré un taux d'incidence de tests positifs subséquents de $14 \%$ ( $n=87 / 619$ cas).

- Les cas de tests positifs subséquents dans cette étude étaient des patients significativement plus jeunes que dans les cas de tests négatifs subséquents. La répartition par sexe ne diffère pas entre les groupes.

Limitations :

- Il n'a pas été mentionné si les cas de patients sortis de l'hôpital impliqués dans l'étude étaient représentatifs des cas hospitalisés.

- Les tests sérologiques n'ont été effectués que pour 288 des 619 cas de l'étude et leurs critères de sélection n'ont pas été mentionnés.

- Les cas de tests positifs subséquents et de tests négatifs subséquents ne différaient pas dans les taux de tests positifs pour les anticorps lgG (99\% et $98 \%$, respectivement). Les taux sériques d'anticorps lgG ne différaient pas non plus entre les groupes, à quelque moment que ce soit après le début de la maladie.

- Les cas de tests positifs subséquents et de tests négatifs subséquents ne différaient pas dans les taux de tests positifs pour les anticorps IgM (37\% et $50 \%$, respectivement). Les taux sériques d'anticorps IgM différaient entre les groupes à différents moments après l'apparition de la maladie : à la semaine 3, les cas de tests positifs subséquents présentaient des niveaux d'lgM significativement plus élevés, tandis qu'aux semaines 6 à 8 , les cas de tests positifs subséquents présentaient des niveaux d'lgM significativement plus faibles.

- Le taux d'incidence de tests positifs subséquents dans cette étude était de $19 \%$ ( $n=93 / 479$ cas). En outre, 45 (9\%) ont connu plusieurs tests positifs subséquents : deux $(n=32,7 \%)$, trois $(n=9,2 \%)$ ou quatre $(n=4,1 \%)$ tests positifs subséquents

- Les cas de tests positifs subséquents dans cette étude étaient significativement plus jeunes que les cas de tests négatifs subséquents (34 ans comparativement à 45 ans). La répartition par sexe ne diffère pas entre les groupes.

Limitations :

- Il n'a pas été mentionné si les cas sortis de l'hôpital impliqués dans l'étude étaient représentatifs des cas hospitalisés.

- Sur les 504 cas de la cohorte, 25 ont été exclus en raison du manque d'informations dans leur dossier médical.

- Les cas de tests positifs subséquents et de tests négatifs subséquents ne différaient pas dans les taux de tests positifs pour les anticorps lgG (100\% et $99,2 \%$, respectivement). prospective a suivi 182 cas sortis de l'hôpital. La sérologie est l'un des principaux résultats de cette étude, 147 cas ayant été soumis à un test sérologique le $7^{\mathrm{e}}$ et le $14^{\mathrm{e}}$ jour après la sortie de l'hôpital.

- Les cas ont été suivis pendant 14 jours. Les patients ont été suivis pendant la quarantaine obligatoire de 14 jours suivant la sortie de l'hôpital et ont été testés à nouveau aux jours 7 et 14 de la quarantaine.
- Les cas de tests positifs subséquents et de tests négatifs subséquents ne différaient pas dans les taux de tests positifs pour les anticorps IgM $(71,4 \%$ pour les deux).

- Le taux d'incident de tests positifs subséquents dans cette étude était de $11 \%$ (20/182 cas).

- Les cas de tests positifs subséquents n'étaient pas significativement plus jeunes dans cette étude; cependant, les cas de moins de 18 ans étaient surreprésentés dans le groupe de patients ayant obtenu un test positif subséquent. La répartition par sexe ne diffère pas entre les groupes.

Limitations :

- Les critères de sélection de la population concernée par l'étude n'ont pas été précisés.

- Le test sérologique ayant été effectué sur une base volontaire, seul un sous-ensemble a eu des résultats sérologiques (14 cas de tests positifs subséquents et 133 cas de tests négatifs subséquents). Aucune information n'a été fournie sur les différences entre ceux qui ont effectué le test sérologique et ceux qui ne l'ont pas fait. 


\section{Annexe A : Caractéristiques sérologiques des cas de maladie à coronavirus 2019 hospitalisés qui ont eu un nouveau test d'amplification en chaîne par polymérase par transcription inverse positif par rapport à ceux dont le nouveau test était négatif (suite)}

\begin{tabular}{|c|c|c|}
\hline $\begin{array}{l}\text { Description de } \\
\text { l'étude } \\
\text { (référence et type, } \\
\text { lieu et dates de } \\
\text { l'étude) }\end{array}$ & Détails du rapport & Principales conclusions et limites \\
\hline $\begin{array}{l}\text { Zhu et al., } 2020 \text { (10) } \\
\text { Étude de cohorte } \\
\text { rétrospective } \\
\text { Zhejiang, Chine } \\
\text { Avant le } 2 \text { avril } 2020\end{array}$ & $\begin{array}{l}\text { - Cette étude de cohorte } \\
\text { rétrospective a suivi } 98 \text { cas de } \\
\text { patients sortis de l'hôpital. La } \\
\text { sérologie faisait partie d'un } \\
\text { large éventail de facteurs } \\
\text { examinés, les tests mesurant } \\
\text { les changements temporels } \\
\text { des niveaux d'anticorps. Le } \\
\text { calendrier exact des tests n'est } \\
\text { pas précisé. } \\
\text { Les cas ont été suivis pendant } \\
\text { au moins } 17 \text { jours après la } \\
\text { sortie de l'hôpital, le délai } \\
\text { moyen avant le nouveau test } \\
\text { positif étant de } 7 \text { jours. }\end{array}$ & $\begin{array}{l}\text { - Dans cette étude, } 35,5 \% \text { des cas de tests positifs subséquents ont été testés } \\
\text { positifs à la fois pour les anticorps lgG et lgM, comparativement }<8,6 \% \\
\text { dans les cas de tests négatifs subséquents. } 58.8 \% \text { des cas de tests positifs } \\
\text { subséquents ont été testés positifs pour les anticorps lgG et négatifs pour les lgM, } \\
\text { comparativement à } 44,4 \% \text { dans les cas de tests négatifs subséquents. Deux cas } \\
\text { de tests positifs subséquents et un cas de test négatif subséquent ont été testés } \\
\text { négatifs pour les anticorps lgG et lgM. Les groupes n'étaient pas significativement } \\
\text { différents. } \\
\text { - Le taux d'incident de tests positifs subséquents était de } 17 \% \text { (17/98 cas). } \\
\text { - Ni l'âge ni le sexe ne différaient entre les cas de tests positifs subséquents et de } \\
\text { tests négatifs subséquents. } \\
\text { Limitations: } \\
\text { - Seuls les patients rétablis ont été suivis. II n'a pas été précisé s'ils étaient } \\
\text { représentatifs des patients hospitalisés. }\end{array}$ \\
\hline $\begin{array}{l}\text { Hu et al., } 2020 \text { (9) } \\
\text { Étude de cohorte } \\
\text { rétrospective } \\
\text { Chongquin, Chine } \\
\text { Du } 23 \text { janvier au } 3 \text { mars } \\
2020\end{array}$ & $\begin{array}{l}\text { - Cette étude a suivi } 221 \text { cas } \\
\text { de patients hospitalisés. La } \\
\text { sérologie était le principal } \\
\text { critère d'évaluation, les } \\
\text { échantillons de sérum étant } \\
\text { prélevés tous les } 3 \text { jours après } \\
\text { l'apparition des symptômes. } \\
\text { - Au total, } 74 \text { cas ont reçu leur } \\
\text { congé et ont été suivis pendant } \\
\text { la période de quarantaine de } \\
14 \text { jours. }\end{array}$ & $\begin{array}{l}\text { - Les cas ayant obtenu un test positif subséquent avaient des taux d'lgG après la } \\
\text { sortie de l'hôpital de } 8,94 \text { en moyenne, comparativement à } 20,19 \text { dans les cas de } \\
\text { tests négatifs subséquents, ce qui est significativement plus faible dans le groupe } \\
\text { des tests positifs subséquents. Les niveaux sont exprimés sous forme de rapport } \\
\text { entre le signal de chimioluminescence et la valeur seuil ( } \mathrm{S} / \mathrm{CO}) \text {. } \\
\text { - Les cas de tests positifs subséquents et de tests négatifs subséquents n'avaient } \\
\text { pas de taux d'IgM significativement différents après la sortie de l'hôpital } \\
\text { (0,90 } \mathrm{S} / \mathrm{CO} \text { comparativement à } 1,39 \mathrm{~S} / \mathrm{CO} \text {, respectivement) } \\
\text { - Cette étude rapporte le taux d'incidence de tests positifs subséquents le plus } \\
\text { élevé des dix études ( } \mathrm{n}=39 / 74 \text {, soit } 52,7 \% \text { ). Aucun délai moyen pour obtenir un } \\
\text { test positif subséquent n'a été indiqué. } \\
\text { - Aucune différence d'âge/sexe entre les cas de tests positifs subséquents et de } \\
\text { tests négatifs subséquents n'a été signalée. } \\
\text { Limitations: } \\
\text { - Seuls } 74 \text { cas de patients ont reçu leur congé et ont été suivis pendant la période } \\
\text { de quarantaine de } 14 \text { jours. II n'a pas été précisé s'ils étaient représentatifs des } \\
\text { patients hospitalisés. }\end{array}$ \\
\hline $\begin{array}{l}\text { Zou et al., } 2020 \text { (11) } \\
\text { Étude de cohorte } \\
\text { rétrospective } \\
\text { Wuhan, Chine } \\
\text { Du 1er janvier au } 10 \\
\text { mars } 2020\end{array}$ & $\begin{array}{l}\text { - Cette étude a suivi } 257 \text { cas de } \\
\text { patients hospitalisés. } \\
\text { - La sérologie n'était pas le } \\
\text { principal résultat de l'étude. On } \\
\text { ne sait pas pendant combien } \\
\text { de temps les patients ont été } \\
\text { suivis ni quand ils ont subi des } \\
\text { tests sérologiques. }\end{array}$ & $\begin{array}{l}\text { - Les cas de tests positifs subséquents et de tests négatifs subséquents ne } \\
\text { différaient pas dans les taux de tests positifs pour les anticorps lgG ( } 94,4 \% \text { et } \\
85,1 \% \text {, respectivement). } \\
\text { - Les cas de tests positifs subséquents et de tests négatifs subséquents ne } \\
\text { différaient pas dans les taux de tests positifs pour les anticorps lgM ( } 52,8 \% \text { et } \\
58,8 \% \text {, respectivement). } \\
\text { - Le taux d'incidence de tests positifs subséquents dans cette étude était de } 20,6 \% \\
\text { ( } \mathrm{n}=53 / 257 \text { cas). } \\
\text { - Ni l'âge ni le sexe ne différaient entre les cas de tests positifs subséquents et de } \\
\text { tests négatifs subséquents. } \\
\text { - On ne sait pas pendant combien de temps les cas ont été suivis, mais on dit qu'ils } \\
\text { ont obtenu un test positif subséquent en moyenne } 4,6 \text { jours après leur sortie de } \\
\text { l'hôpital. } \\
\text { - L'objectif de cette étude était de comparer les taux de tests positifs subséquents } \\
\text { pour les cas ayant obtenu deux tests PCR négatifs subséquents par rapport à trois } \\
\text { négatifs subséquents pour obtenir leur congé de l'hôpital. } 20.6 \% \text { des cas ayant } \\
\text { subi deux tests négatifs présentent un test positif subséquent, comparativement à } \\
\text { seulement } 5,4 \% \text { des cas ayant obtenu trois tests négatifs. } \\
\text { Limitations : } \\
\text { - Seul un sous-ensemble avait des résultats sérologiques (36 cas de tests positifs } \\
\text { subséquents et } 114 \text { cas de tests négatifs subséquents). Les informations sur leur } \\
\text { différence avec ceux qui n'ont pas subi de test n'ont pas été mentionnées. }\end{array}$ \\
\hline
\end{tabular}




\section{Annexe A : Caractéristiques sérologiques des cas de maladie à coronavirus 2019 hospitalisés qui ont eu un nouveau test d'amplification en chaîne par polymérase par transcription inverse positif par rapport à ceux dont le nouveau test était négatif (suite)}

\section{Description de} l'étude

(référence et type,

lieu et dates de l'étude)

\section{Détails du rapport}

\section{Principales conclusions et limites}

\section{Huang et al., 2020 (13)}

Étude de cohorte - il n'est pas mentionné si elle est prospective ou rétrospective

Shenzhen, Chine

Du 11 janvier au 23 avri 2020
- Cette étude a suivi 414 cas de patients hospitalisés. La sérologie faisait partie d'un large éventail de facteurs examinés. 154 cas ont subi un test sérologique à la sortie de l'hôpital.

- Les cas ont été suivis pendant quatre semaines après leur sortie. Ils ont reçu un nouveau test positif au dixième jour en moyenne, les tests RT-PCR étant effectués tous les 3 à 5 jours.

- Les cas de tests positifs subséquents et de tests négatifs subséquents ne différaient pas dans les taux de tests positifs pour les anticorps lgG $(100 \%$ et $99,1 \%$, respectivement).

- Les cas de tests positifs subséquents et de tests négatifs subséquents ne différaient pas dans les taux de tests positifs pour les anticorps IgM $(75,0 \%$ et $48,2 \%$, respectivement).

- Le taux d'incidence de tests positifs subséquents dans cette étude était de $16,7 \%$ ( $n=69 / 414$ cas).

- Les cas de tests positifs subséquents dans cette étude étaient des patients significativement plus jeunes que dans les cas de tests négatifs subséquents. La répartition par sexe ne diffère pas entre les groupes.

Limitations :

- II n'a pas été précisé si la population étudiée était représentative des patients hospitalisés.

Liu et al., 2020 (12)

Étude de cohorte - il n'est pas mentionné si elle est prospective ou rétrospective

Wuhan, Chine

Du $1^{\text {er }}$ au 13 mars 2020

Étude cas-témoin Wuhan, Chine Du 10 février au 31 mars 2020
- Cette étude a suivi 150 cas de sortie de l'hôpital.

- La sérologie était le principal critère d'évaluation, mais ni le moment de la sérologie ni la durée du suivi n'ont été indiqués. Les tests effectués à différents moments après le congé de l'hôpital peuvent avoir des incidences sur les résultats.
- Les cas de tests positifs subséquents et de tests négatifs subséquents ne différaient pas dans les taux de tests positifs pour les anticorps lgG (100\% et $90,6 \%$, respectivement). Les taux sériques d'anticorps IgG ne différaient pas non plus entre les groupes ( $243 \mathrm{AU} / \mathrm{mL}$ et $185 \mathrm{AU} / \mathrm{mL}$, respectivement).

- Les cas de tests positifs subséquents et de tests négatifs subséquents ne différaient pas dans les taux de tests positifs pour les anticorps IgM (45,5\% et $47,5 \%$, respectivement). Les taux sériques d'anticorps lgM ne différaient pas non plus entre les groupes $(9,6 \mathrm{AU} / \mathrm{mL}$ et $8,9 \mathrm{AU} / \mathrm{mL}$, respectivement).

- Le taux d'incident de tests positifs subséquents dans cette étude était de 7,3\% (11/150 cas).

- Ni l'âge ni le sexe ne différaient entre les cas de tests positifs subséquents et de tests négatifs subséquents.

Limitations :

- Seuls les patients convalescents ont été suivis. II n'a pas été précisé s'ils étaient représentatifs des patients hospitalisés.

- Le calendrier des tests sérologiques n'est pas clair.

- Les cas de patients ayant obtenu un test positif subséquent avaient des taux d'lgG de 78,53 AU/mL en moyenne, comparativement $<147,85 \mathrm{AU} / \mathrm{mL}$ dans les cas de tests négatifs subséquents, ce qui est significativement différent.

- Les cas de tests positifs subséquents présentaient des taux d'IgM de 13,69 AU/mL en moyenne, comparativement $<68,10 \mathrm{AU} / \mathrm{mL}$ chez les cas de patient ayant reçu un test négatif subséquent, ce qui est significativement différent.

- Rapporte le taux d'incidence de tests positifs subséquents le plus faible des dix études ( $n=2 / 107$, soit $1,9 \%$ ) de la cohorte dans un seul hôpital. Quinze cas provenant de plusieurs sites ont été comparés à 107 témoins.

- Les cas de tests positifs subséquents se sont avérés être significativement plus jeunes que les cas de tests négatifs subséquents (43 ans par rapport à 60 ans). II n'y avait pas de différence significative entre le sexe des cas de tests positifs subséquents et de tests négatifs subséquents.

Limitations :

- L'âge et le sexe n'étaient pas appariés entre les cas et les témoins.

- Le moment où les tests sérologiques ont été effectués n'était pas clair. 
Annexe B : Évaluation, à l'aide de l'outil d'évaluation du risque de biais de l'échelle de Newcastle-Ottawa, de neuf études publiées jusqu'en septembre 2020, comparant les indicateurs de la réponse immunitaire des cas de maladie à coronavirus 2019 chez des patients ayant obtenu leur congé de l'hôpital, qui ont obtenu un résultat positif subséquent au test de dépistage à l'aide d'une réaction en chaîne par polymérase après transcription inverse, à ceux qui n'ont pas obtenu de résultat positif à un test subséquent

\begin{tabular}{|c|c|c|c|}
\hline Étude & $\begin{array}{l}\text { Sélection } \\
(\text { score }=4)\end{array}$ & $\begin{array}{l}\text { Comparabilité } \\
\text { (score = 2) }\end{array}$ & $\begin{array}{l}\text { Exposition/résultat } \\
\text { (score }=3)\end{array}$ \\
\hline $\begin{array}{l}\text { Caractérisation clinique, immunologique et virologique des patients du } \\
\text { COVID-19 dont le test de RT-PCR s'est à nouveau révélé positif pour le } \\
\text { SRAS-CoV-2 (6). }\end{array}$ & 2 & 0 & 3 \\
\hline $\begin{array}{l}\text { Récurrence d'ARN viral SRAS-CoV-2 positif chez les patients rétablis de la } \\
\text { COVID-19 pendant l'observation d'isolement médical (7). }\end{array}$ & 2 & 0 & 1 \\
\hline $\begin{array}{l}\text { Niveau d'ARN viral, réponses immunitaires sériques et risque de transmission } \\
\text { chez les patients atteints de COVID-19 ayant obtenu leur congé de l'hôpital } \\
\text { et obtenant des résultats positifs récurrents au test d'ARN du SRAS-CoV-2: } \\
\text { une étude de cohorte observationnelle fondée sur la population (8). }\end{array}$ & 2 & 0 & 2 \\
\hline $\begin{array}{l}\text { Caractéristiques cliniques des patients convalescents de la COVID-19 avec } \\
\text { nouvelle détection d'acide nucléique positif (10). }\end{array}$ & 3 & 0 & 2 \\
\hline $\begin{array}{l}\text { La production d'anticorps contre le SRAS-CoV-2 et son implication } \\
\text { clinique (9). }\end{array}$ & 3 & 0 & 1 \\
\hline $\begin{array}{l}\text { La question des patients positifs récurrents qui se sont rétablis de la } \\
\text { COVID-19 selon les critères de congé actuels : enquête sur les patients de } \\
\text { plusieurs institutions médicales à Wuhan, en Chine (11). }\end{array}$ & 3 & 0 & 1 \\
\hline $\begin{array}{l}\text { Récurrence de la positivité du SRAS-CoV-2 chez les patients infectés } \\
\text { et rétablis : Une expérience sur la COVID-19 dans un seul centre et ses } \\
\text { implications potentielles (13). }\end{array}$ & 4 & 0 & 2 \\
\hline $\begin{array}{l}\text { SRAS-CoV-2 positif récurrent - le certificat d'immunité pourrait ne pas être } \\
\text { valide (12). }\end{array}$ & 3 & 0 & 2 \\
\hline $\begin{array}{l}\text { Caractéristiques cliniques de la maladie à coronavirus } 2019 \text { récurrente et } \\
\text { positive après un congé en raison d'un rétablissement : analyse rétrospective } \\
\text { de } 15 \text { cas à Wuhan en Chine (14). }\end{array}$ & 3 & 0 & 3 \\
\hline
\end{tabular}

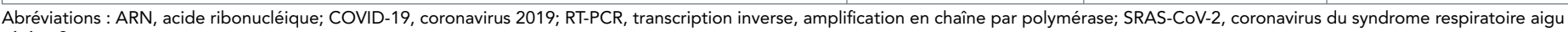
sévère 2 